\title{
Management of Automotive Engine Based on Stable Fuzzy Technique with Parallel Sliding Mode Optimization
}

\author{
Mansour Bazregar \\ Industrial Electrical and Electronic Engineering SanatkadeheSabze Pasargad, CO (S.S.P. Co), Shiraz, Iran \\ E-mail:SSP.ROBOTIC@yahoo.com
}

Farzin Piltan

Senior Researcher at Research and Development Unit, SanatkadeheSabze Pasargad Company, (S.S.P. Co), Shiraz, Iran E-mail:Piltan_f@iranssp.com; WWW.IRANSSP.COM

\author{
Mehdi Akbari \\ Industrial Electrical and Electronic Engineering SanatkadeheSabze Pasargad, CO (S.S.P. Co), Shiraz, Iran \\ E-mail: SSP.ROBOTIC@yahoo.com \\ Mojdeh Piran \\ Industrial Electrical and Electronic Engineering SanatkadeheSabze Pasargad, CO (S.S.P. Co), Shiraz, Iran \\ E-mail:SSP.ROBOTIC@yahoo.com
}

\begin{abstract}
Both fuzzy logic and sliding mode can compensate the steady-state error of proportionalderivative (PD) method. This paper presents parallel sliding mode optimization for fuzzy PD management. The asymptotic stability of fuzzy PD management with first-order sliding mode optimization in the parallel structure is proven. For the parallel structure, the finite time convergence with a super-twisting second-order sliding-mode is guaranteed.
\end{abstract}

Index Terms - Fuzzy Logic Method, Linear PD Method, Sliding Mode Methodology, Parallel Method

\section{Introduction}

The internal combustion (IC) engine is designed to produce power from the energy that is contained in its fuel. More specifically, its fuel contains chemical energy and together with air, this mixture is burned to output mechanical power. There are various types of fuels which can be used in IC engines namely; petroleum, diesel, bio-fuels, and hydrogen [1]. Modeling of an entire IC engine is a very important and complicated process because engines are nonlinear, multi inputs-multi outputs (MIMO) and time variant. One of the significant challenges in control manage algorithms is a linear behavior controller design for nonlinear systems (e.g., IC engine). Some of IC engines which work in industrial processes are controlled by linear PD management, proportional-integral-derivative
(PID) method, but the design of linear management for IC engines is extremely difficult because they are hardly nonlinear and uncertain $[1-2,6]$. To reduce the above challenges, the nonlinear robust management is used to compensate the linear control of IC engine.

Controller is a device which can sense information from linear or nonlinear system (e.g., IC engine) to improve the systems performance [3]. The main targets in designing control systems are stability, good disturbance rejection, and small tracking error[5]. Several industrial IC engines are controlled by linear methodologies (e.g., Proportional-Derivative (PD) management, Proportional- Integral (PI) management or Proportional- Integral-Derivative (PID) management), but when IC engine works in various situations and have uncertainty in dynamic models this technique has limitations. From the control point of view, uncertainty is divided into two main groups: uncertainty in unstructured inputs (e.g., noise, disturbance) and uncertainty in structure dynamics (e.g., payload, parameter variations). In some applications IC engines are used in an unknown and unstructured environment, therefore strong mathematical tools used in new control methodologies to design fuzzy PD controller based on sliding mode compensation to have an acceptable performance (e.g., minimum error, good trajectory, disturbance rejection) [1-7].

Fuzzy-logic aims to provide an approximate but effective means of describing the behavior of systems that are not easy to describe precisely, and which are complex or ill-defined [7-11, 22]. It is based on the 
assumption that, in contrast to Boolean logic, a statement can be partially true (or false) [12-21, 23-33]. For example, the expression (I live near SSP.Co) where the fuzzy value (near) applied to the fuzzy variable (distance), in addition to being imprecise, is subject to interpretation. The essence of fuzzy control is to build a model of human expert who is capable of controlling the plant without thinking in terms of its mathematical model. As opposed to conventional control approaches where the focus is on constructing a controller described by differential equations, in fuzzy control the focus is on gaining an intuitive understanding (heuristic data) of how to best control the process [28], and then load this data into the control system [34-49].

Sliding mode control (SMC) is obtained by means of injecting a nonlinear discontinuous term. This discontinuous term is the one which enables the system to reject disturbances and also some classes of mismatches between the actual system and the model used for design [12, 36-69]. These standard SMCs are robust with respect to internal and external perturbations, but they are restricted to the case in which the output relative degree is one. Besides, the high frequency switching that produces the sliding mode may cause chattering effect. The tracking error of SMC converges to zero if its gain is bigger than the upper bound of the unknown nonlinear function. Boundary layer SMC can assure no chattering happens when tracking error is less than; but the tracking error converges to; it is not asymptotically stable [13]. A new generation of SMC using second-order sliding-mode has been recently developed by [15] and [16]. This higher order SMC preserves the features of the first order SMC and improves it in eliminating the chattering and fast convergence [33-69].

Normal combinations of PD control with fuzzy logic (PD+FL) and sliding mode (PD+SMC) are to apply these three controllers at the same time [17], while FLC compensates the control error, SMC reduces the remain error of fuzzy PD such that the final tracking error is asymptotically stable [18]. The chattering is eliminating, because PD+SMC and PD+FL work parallel. In this paper, the asymptotic stability of PD control with parallel fuzzy logic and the first-order sliding mode compensation is proposed (PD+SMC+FL). The fuzzy PD is used to approximate the nonlinear plant. A dead one algorithm is applied for the fuzzy PD control. After the regulation error enter converges to the dead-zone, a super-twisting second-order sliding-mode is used to guarantee finite time convergence of the whole control (PD+FL+SMC). By means of a Lyapunov approach, we prove that this type of control can ensure finite time convergence and less chattering than SMC and SMC+FL [33-39].

This paper is organized as follows; second part focuses on the modeling dynamic formulation based on Lagrange methodology, fuzzy logic methodology and sliding mode controller to have a robust control. Third part is focused on the methodology which can be used to reduce the error, increase the performance quality and increase the robustness and stability. Simulation result and discussion is illustrated in forth part which based on trajectory following and disturbance rejection. The last part focuses on the conclusion and compare between this method and the other ones.

\section{Theorem:}

\section{- IC Engine's Dynamic:}

Dynamic modeling of IC engine is used to describe the nonlinear behavior of IC engine, design of model based controller such as pure variable structure controller based on nonlinear dynamic equations, and for simulation. The dynamic modeling describes the relationship between fuel to air ratio to PFI and DI and also it can be used to describe the particular dynamic effects (e.g., motor pressure, angular speed, mass of air in cylinder, and the other parameters) to behavior of system[1].

The equation of an IC engine governed by the following equation $[1,4,25,29]$ :

$$
\begin{aligned}
& {\left[\begin{array}{c}
\mathrm{PFI} \\
\mathrm{DI}
\end{array}\right]=\left[\begin{array}{ll}
\dot{\mathrm{M}}_{\text {air } 11} & \dot{\mathrm{M}}_{\text {air } 12} \\
\dot{\mathrm{M}}_{\text {air } 21} & \dot{\mathrm{M}}_{\text {air }_{22}}
\end{array}\right]\left[\begin{array}{c}
\mathrm{FR} \\
\ddot{\alpha}_{\mathrm{I}}
\end{array}\right]+} \\
& {\left[\begin{array}{l}
\mathrm{P}_{\text {motor }_{1}} \\
\mathrm{P}_{\text {motor }_{2}}
\end{array}\right]\left[\begin{array}{ll}
\mathrm{FR} & \dot{\alpha}_{\mathrm{I}}
\end{array}\right]+\left[\begin{array}{ll}
\mathrm{N}_{11} & \mathrm{~N}_{12} \\
\mathrm{~N}_{21} & \mathrm{~N}_{22}
\end{array}\right] \times\left[\begin{array}{c}
\dot{F R}^{2} \\
\dot{\alpha}_{\mathrm{I}}
\end{array}\right]^{2}+} \\
& {\left[\begin{array}{l}
\mathrm{M}_{\mathrm{a}_{1}} \\
\mathrm{M}_{\mathrm{a}_{2}}
\end{array}\right]}
\end{aligned}
$$

Where $P F I$ is port fuel injector, $D I$ is direct injector, $\dot{\mathbf{M}}_{\text {air }}$ is a symmetric and positive define mass of air matrix, $\mathbf{P}_{\text {motor }}$ is the pressure of motor, $N$ is engine angular speed and $\mathbf{M}_{\mathbf{a}}$ is matrix mass of air in cylinder. Fuel ratio and exhaust angle are calculated by [25, 29]:

$$
\begin{aligned}
& {\left[\begin{array}{c}
F \ddot{R}_{a} \\
\ddot{\alpha}_{I_{a}}
\end{array}\right]=\left[\begin{array}{ll}
\dot{M}_{\text {air }_{11}} & \dot{M}_{\text {air }_{12}} \\
\dot{M}_{\text {air } 21} & \dot{M}_{\text {air } 22}
\end{array}\right]^{-1}\left\{\left[\begin{array}{c}
P F I \\
D I
\end{array}\right]-\right.} \\
& \left\{\left[\begin{array}{c}
P_{\text {motor }_{1}} \\
P_{\text {motor }_{2}}
\end{array}\right]\left[\begin{array}{ll}
F R & \dot{\alpha}_{I_{a}}
\end{array}\right]+\left[\begin{array}{ll}
N_{11} & N_{12} \\
N_{21} & N_{22}
\end{array}\right] \times\right. \\
& \left.\left[\begin{array}{c}
F \dot{R}_{a} \\
\dot{\alpha}_{I_{a}}
\end{array}\right]^{2}+\left[\begin{array}{c}
M_{a_{1}} \\
M_{a_{2}}
\end{array}\right]\right\}
\end{aligned}
$$

The above target equivalence ratio calculation will be combined with fuel ratio calculation that will be used for controller design purpose.

\subsection{Model free Control Technique}

The model-free control strategy is based on the assumption that the joints of the manipulators are all independent and the system can be decoupled into a group of single-axis control systems [18-23]. Therefore, the kinematic control method always results in a group 
of individual controllers, each for an active joint of the manipulator. With the independent joint assumption, no a priori knowledge of IC engine dynamics is needed in the kinematic controller design, so the complex computation of its dynamics can be avoided and the controller design can be greatly simplified. This is suitable for real-time control applications when powerful processors, which can execute complex algorithms rapidly, are not accessible. However, since joints coupling is neglected, control performance degrades as operating speed increases and a manipulator controlled in this way is only appropriate for relatively slow motion [33]. The fast motion requirement results in even higher dynamic coupling between the various robot joints, which cannot be compensated for by a standard robot controller such as PD [21], and hence model-based control becomes the alternative. Based on above discussion;

$$
\begin{aligned}
& e_{1}(t)=F R_{\text {desired }}(t)-F R_{\text {actual }}(t) \\
& P F I_{\alpha}=K_{p_{a}} e_{1}+K_{V_{a}} e_{1}
\end{aligned}
$$

\subsection{Sliding Mode Controller}

Consider a nonlinear single input dynamic system is defined by [6]:

$$
x^{(n)}=f(\vec{x})+b(\vec{x}) u
$$

Where $\mathrm{u}$ is the vector of control input, $\boldsymbol{x}^{(\boldsymbol{n})}$ is the $\boldsymbol{n}^{\boldsymbol{t h}}$ derivation of $x, x=\left[x, \dot{x}, \ddot{x}, \ldots, x^{(n-1)}\right]^{T}$ is the state vector, $\boldsymbol{f}(\boldsymbol{x})$ is unknown or uncertainty, and $\boldsymbol{b}(\boldsymbol{x})$ is of known sign function. The main goal to design this management is train to the desired state; $\boldsymbol{x}_{\boldsymbol{d}}=$ $\left[x_{d}, \dot{x}_{d}, \ddot{x}_{d}, \ldots, x_{d}{ }^{(n-1)}\right]^{T}$, and trucking error vector is defined by [6]:

$$
\widetilde{x}=x-x_{d}=\left[\widetilde{x}, \ldots, \widetilde{x}^{(n-1)}\right]^{T}
$$

A time-varying sliding surface $\boldsymbol{s}(\boldsymbol{x}, \boldsymbol{t})$ in the state space $\boldsymbol{R}^{\boldsymbol{n}}$ is given by [6]:

$$
s(x, t)=\left(\frac{d}{d t}+\lambda\right)^{n-1} \tilde{x}=0
$$

where $\lambda$ is the positive constant. To further penalize tracking error, integral part can be used in sliding surface part as follows [6]:

$$
\boldsymbol{s}(\boldsymbol{x}, \boldsymbol{t})=\left(\frac{d}{d t}+\lambda\right)^{n-1}\left(\int_{0}^{t} \tilde{\boldsymbol{x}} \boldsymbol{d t}\right)=\mathbf{0}
$$

The main target in this methodology is kept the sliding surface slope $\boldsymbol{s}(\boldsymbol{x}, \boldsymbol{t})$ near to the zero. Therefore, one of the common strategies is to find input $\boldsymbol{U}$ outside of $s(x, t)[6]$.

$$
\frac{1}{2} \frac{d}{d t} s^{2}(x, t) \leq-\zeta|s(x, t)|
$$

where $\zeta$ is positive constant.

$$
\text { If } \mathbf{S}(\mathbf{0})>\mathbf{0} \rightarrow \frac{\mathrm{d}}{\mathrm{dt}} \mathbf{S}(\mathbf{t}) \leq-\zeta
$$

To eliminate the derivative term, it is used an integral term from $\mathrm{t}=0$ to $\mathrm{t}=\boldsymbol{t}_{\text {reach }}$

$$
\begin{aligned}
& \int_{t=0}^{t=t_{\text {reach }}} \frac{d}{d t} S(t) \leq-\int_{t=0}^{t=t_{\text {reach }}} \eta \rightarrow \\
& S\left(t_{\text {reach }}\right)-S(0) \leq-\zeta\left(t_{\text {reach }}-0\right)
\end{aligned}
$$

Where $t_{\text {reach }}$ is the time that trajectories reach to the sliding surface so, suppose $\mathrm{S}\left(t_{\text {reach }}=0\right)$ defined as;

$$
0-S(0) \leq-\eta\left(t_{\text {reach }}\right) \rightarrow t_{\text {reach }} \leq \frac{S(0)}{\zeta}
$$

and

$$
\begin{aligned}
& \text { if } \boldsymbol{S}(0)<0 \rightarrow 0-S(0) \leq-\eta\left(\boldsymbol{t}_{\text {reach }}\right) \rightarrow \\
& \boldsymbol{S}(\mathbf{0}) \leq-\zeta\left(\boldsymbol{t}_{\text {reach }}\right) \rightarrow \boldsymbol{t}_{\text {reach }} \leq \frac{|\boldsymbol{S}(\mathbf{0})|}{\eta}
\end{aligned}
$$

Equation (13) guarantees time to reach the sliding surface is smaller than $\frac{|\boldsymbol{S}(\mathbf{0})|}{\zeta}$ since the trajectories are outside of $S(t)$.

$$
\text { if } S_{t_{\text {reach }}}=S(0) \rightarrow \operatorname{error}\left(x-x_{d}\right)=0
$$

suppose $\mathrm{S}$ is defined as

$$
\begin{aligned}
s(x, t)=\left(\frac{d}{d t}+\lambda\right) & \tilde{x} \\
= & \left(\dot{\mathbf{x}}-\dot{\mathbf{x}}_{\mathbf{d}}\right)+\lambda\left(\mathbf{x}-\mathbf{x}_{\mathbf{d}}\right)
\end{aligned}
$$

The derivation of $\mathrm{S}$, namely, $\dot{S}$ can be calculated as the following;

$$
\dot{S}=\left(\ddot{\mathbf{x}}-\ddot{\mathbf{x}}_{\mathbf{d}}\right)+\lambda\left(\dot{\mathbf{x}}-\dot{\mathbf{x}}_{\mathbf{d}}\right)
$$

suppose the second order system is defined as;

$$
\begin{array}{r}
\ddot{x}=f+u \rightarrow \dot{S}=f+U-\ddot{x}_{d} \\
+\lambda\left(\dot{\mathbf{x}}-\dot{\mathbf{x}}_{\mathrm{d}}\right)
\end{array}
$$

Where $\boldsymbol{f}$ is the dynamic uncertain, and also since $S=$ 0 and $\dot{S}=0$, to have the best approximation, $\widehat{\boldsymbol{U}}$ is defined as

$$
\widehat{U}=-\widehat{f}+\ddot{x}_{d}-\lambda\left(\dot{\mathbf{x}}-\dot{\mathbf{x}}_{\mathbf{d}}\right)
$$

A simple solution to get the sliding condition when the dynamic parameters have uncertainty is the switching control law [12-13]:

$$
U_{d i s}=\widehat{U}-K(\vec{x}, t) \cdot \operatorname{sgn}(s)
$$



6]

where the switching function $\mathbf{s g n}(\mathbf{S})$ is defined as [1,

$$
\operatorname{sgn}(s)= \begin{cases}1 & s>0 \\ -1 & s<0 \\ 0 & s=0\end{cases}
$$

and the $\boldsymbol{K}(\overrightarrow{\boldsymbol{x}}, \boldsymbol{t})$ is the positive constant. Suppose by (9) the following equation can be written as,

$$
\begin{aligned}
& \frac{1}{2} \frac{d}{d t} S^{2}(x, t)=\dot{S} \cdot S=[f-\widehat{f}-K \operatorname{sgn}(s)] \\
& S=(f-\widehat{f}) \cdot S-K|S|
\end{aligned}
$$

and if the equation (13) instead of (12) the sliding surface can be calculated as

$$
\begin{aligned}
& s(x, t)=\left(\frac{d}{d t}+\lambda\right)^{2}\left(\int_{0}^{t} \widetilde{x} d t\right)=\left(\dot{x}-\dot{x}_{d}\right)+ \\
& 2 \lambda\left(\dot{x}-\dot{x}_{d}\right)-\lambda^{2}\left(x-x_{d}\right)
\end{aligned}
$$

in this method the approximation of $\boldsymbol{U}$ is computed as [6]

$$
\widehat{U}=-\widehat{f}+\ddot{x}_{d}-2 \lambda\left(\dot{\mathbf{x}}-\dot{\mathbf{x}}_{\mathbf{d}}\right)+\lambda^{2}\left(\mathbf{x}-\mathbf{x}_{\mathbf{d}}\right)
$$

Based on above discussion, the sliding mode control law for a multi degrees of freedom IC engine is written as $[1,6]$ :

$$
P F I=P F I_{e q}+P F I_{d i s}
$$

Where, the model-based component $\boldsymbol{P F I _ { e q }}$ is the nominal dynamics of systems calculated as follows [1]:

$$
\begin{aligned}
& P F I_{e q}= \\
& {\left[[ \begin{array} { l l } 
{ \dot { M } _ { \text { air } } 1 1 } & { \dot { M } _ { \text { air } } 1 2 } \\
{ \dot { M } _ { \text { air } } 2 1 } & { \dot { M } _ { \text { air } 2 2 } }
\end{array} ] ^ { - 1 } \left(\left[\begin{array}{l}
\mathrm{P}_{\text {motor }_{1}} \\
\mathrm{P}_{\text {motor }_{2}}
\end{array}\right]\left[\begin{array}{ll}
\dot{\mathrm{FR}} & \dot{\alpha}_{\mathrm{I}}
\end{array}\right]+\right.\right.} \\
& \left.\left[\begin{array}{ll}
\mathrm{N}_{11} & \mathrm{~N}_{12} \\
\mathrm{~N}_{21} & \mathrm{~N}_{22}
\end{array}\right] \times\left[\begin{array}{c}
\mathrm{FR} \\
\dot{\alpha_{1}}
\end{array}\right]^{2}+\left[\begin{array}{l}
\mathrm{M}_{\mathrm{a}_{1}} \\
\mathrm{M}_{\mathrm{a}_{2}}
\end{array}\right]\right)+ \\
& \dot{\boldsymbol{S}}]\left[\begin{array}{ll}
\dot{\mathrm{M}}_{\text {air }} & \dot{\mathrm{M}}_{\mathrm{air}_{12}} \\
\dot{\mathrm{M}}_{\mathrm{air}_{21}} & \dot{\mathrm{M}}_{\mathrm{air}_{22}}
\end{array}\right]
\end{aligned}
$$

and $\boldsymbol{P F} \boldsymbol{I}_{\text {dis }}$ is computed as [1];

$$
P F I_{\text {dis }}=K \cdot \operatorname{sgn}(S)
$$

By (26) and (25) the sliding mode control of IC engine is calculated as;

$$
\begin{aligned}
& \boldsymbol{P F I}= \\
& {\left[\begin{array}{ll}
\dot{M}_{\text {air }} & \dot{M}_{\text {air }} 12 \\
\dot{M}_{\text {air }} 2 & \dot{M}_{\text {air } 22}
\end{array}\right]^{-1}\left(\left[\begin{array}{l}
\mathrm{P}_{\text {motor }_{1}} \\
\mathrm{P}_{\text {motor }_{2}}
\end{array}\right]\left[\begin{array}{ll}
\mathrm{FR} & \dot{\alpha}_{\mathrm{I}}
\end{array}\right]+\right.} \\
& \left.\left[\begin{array}{ll}
\mathrm{N}_{11} & \mathrm{~N}_{12} \\
\mathrm{~N}_{21} & \mathrm{~N}_{22}
\end{array}\right] \times\left[\begin{array}{c}
\mathrm{FR} \\
\dot{\alpha_{\mathrm{I}}}
\end{array}\right]^{2}+\left[\begin{array}{l}
\mathrm{M}_{\mathrm{a}_{1}} \\
\mathrm{M}_{\mathrm{a}_{2}}
\end{array}\right]\right)+ \\
& \dot{\boldsymbol{S}}]\left[\begin{array}{ll}
\dot{\mathrm{M}}_{\text {air }_{11}} & \dot{\mathrm{M}}_{\text {air }_{12}} \\
\dot{\mathrm{M}}_{\text {air }} & \dot{\mathrm{M}}_{\text {air }_{22}}
\end{array}\right]+\boldsymbol{K} \cdot \operatorname{sgn}(\boldsymbol{S})
\end{aligned}
$$

\subsection{Proof of Stability}

The lyapunov formulation can be written as follows,

$$
\boldsymbol{V}=\frac{\mathbf{1}}{\mathbf{2}} \boldsymbol{S}^{\boldsymbol{T}} \cdot\left[\begin{array}{ll}
\dot{\mathrm{M}}_{\mathrm{air}_{11}} & \dot{\mathrm{M}}_{\mathrm{air}_{12}} \\
\dot{\mathrm{M}}_{\mathrm{air}_{21}} & \dot{\mathrm{M}}_{\mathrm{air}_{22}}
\end{array}\right] . \boldsymbol{S}
$$

the derivation of $V$ can be determined as,

$$
\begin{aligned}
& \dot{\boldsymbol{V}}=\frac{\mathbf{1}}{\mathbf{2}} \boldsymbol{S}^{\boldsymbol{T}} \cdot\left[\begin{array}{ll}
\dot{\mathrm{M}}_{\mathrm{arr}_{11}} & \dot{\mathrm{M}}_{\mathrm{arr}_{12}} \\
\dot{\mathrm{M}}_{\mathrm{arr}_{21}} & \dot{\mathrm{M}}_{\mathrm{arr}_{22}}
\end{array}\right] \cdot \boldsymbol{S}+ \\
& \boldsymbol{S}^{\boldsymbol{T}}\left[\begin{array}{ll}
\dot{\mathrm{M}}_{\mathrm{air}_{11}} & \dot{\mathrm{M}}_{\mathrm{air}_{12}} \\
\dot{\mathrm{M}}_{\mathrm{air}_{21}} & \dot{\mathrm{M}}_{\mathrm{air}_{22}}
\end{array}\right] \dot{\boldsymbol{S}}
\end{aligned}
$$

the dynamic equation of IC engine can be written based on the sliding surface as

$$
\begin{aligned}
& \boldsymbol{M} \dot{\boldsymbol{S}}=-\boldsymbol{V} \boldsymbol{S}+\left[\begin{array}{ll}
\dot{\mathrm{M}}_{\mathrm{air}_{11}} & \dot{\mathrm{M}}_{\mathrm{air}_{12}} \\
\dot{\mathrm{M}}_{\mathrm{air}_{21}} & \dot{\mathrm{M}}_{\mathrm{air}_{22}}
\end{array}\right] \dot{\boldsymbol{S}}+ \\
& \left(\left[\begin{array}{l}
\mathrm{P}_{\text {motor }_{1}} \\
\mathrm{P}_{\text {motor }_{2}}
\end{array}\right]\left[\begin{array}{ll}
\mathrm{FR} & \dot{\alpha}_{\mathrm{I}}
\end{array}\right]+\left[\begin{array}{ll}
\mathrm{N}_{11} & \mathrm{~N}_{12} \\
\mathrm{~N}_{21} & \mathrm{~N}_{22}
\end{array}\right] \times\right. \\
& \left.\left[\begin{array}{c}
\mathrm{FR}^{2} \\
\dot{\alpha_{\mathrm{I}}}
\end{array}\right]^{2}+\left[\begin{array}{l}
\mathrm{M}_{\mathrm{a}_{1}} \\
\mathrm{M}_{\mathrm{a}_{2}}
\end{array}\right]\right)
\end{aligned}
$$

It is assumed that

$$
\begin{aligned}
& \boldsymbol{S}^{\boldsymbol{T}}\left(\left[\begin{array}{ll}
\dot{\mathrm{M}}_{\mathrm{arr}_{11}} & \dot{\mathrm{M}}_{\mathrm{arr}_{12}} \\
\dot{\mathrm{M}}_{\mathrm{arr}_{21}} & \dot{\mathrm{M}}_{\mathrm{arr}_{22}}
\end{array}\right]-\right. \\
& \mathbf{2}\left[\begin{array}{l}
\mathrm{P}_{\text {motor }_{1}} \\
\mathrm{P}_{\text {motor }_{2}}
\end{array}\right]\left[\begin{array}{ll}
\mathrm{FR} & \dot{\mathrm{d}}_{\mathrm{I}}
\end{array}\right]+\left[\begin{array}{ll}
\mathrm{N}_{11} & \mathrm{~N}_{12} \\
\mathrm{~N}_{21} & \mathrm{~N}_{22}
\end{array}\right] \times \\
& \left.\left[\begin{array}{c}
\mathrm{FR} \\
\dot{\alpha_{\mathrm{I}}}
\end{array}\right]^{2}+\left[\begin{array}{l}
\mathrm{M}_{\mathrm{a}_{1}} \\
\mathrm{M}_{\mathrm{a}_{2}}
\end{array}\right]\right) \boldsymbol{S}=\mathbf{0}
\end{aligned}
$$

by substituting (30) in (31)

$$
\begin{aligned}
& \dot{\boldsymbol{V}}=\frac{\mathbf{1}}{\mathbf{2}} \boldsymbol{S}^{\boldsymbol{T}}\left[\begin{array}{ll}
\dot{\mathrm{M}}_{\mathrm{arr}_{11}} & \dot{\mathrm{M}}_{\mathrm{arr}_{12}} \\
\dot{\mathrm{M}}_{\mathrm{arr}} & \dot{\mathrm{M}}_{\mathrm{arr}_{22}}
\end{array}\right] \boldsymbol{S}- \\
& \boldsymbol{S}^{T}\left[\begin{array}{l}
\mathrm{P}_{\text {motor }_{1}} \\
\mathrm{P}_{\text {motor }_{2}}
\end{array}\right]\left[\begin{array}{ll}
\mathrm{FR} & \dot{\alpha}_{\mathrm{I}}
\end{array}\right]+\left[\begin{array}{ll}
\mathrm{N}_{11} & \mathrm{~N}_{12} \\
\mathrm{~N}_{21} & \mathrm{~N}_{22}
\end{array}\right] \times \\
& {\left[\begin{array}{c}
\mathrm{FR}^{2} \\
\dot{\alpha_{\mathrm{I}}}
\end{array}\right]^{2} \boldsymbol{S}+\boldsymbol{S}^{T}\left(\left[\begin{array}{ll}
\dot{\mathrm{M}}_{\mathrm{air}_{11}} & \dot{\mathrm{M}}_{\mathrm{air}_{12}} \\
\dot{\mathrm{M}}_{\mathrm{air}_{21}} & \dot{\mathrm{M}}_{\mathrm{air}_{22}}
\end{array}\right] \dot{\boldsymbol{S}}+\right.} \\
& \left(\left[\begin{array}{l}
\mathrm{P}_{\text {motor }_{1}} \\
\mathrm{P}_{\text {motor }_{2}}
\end{array}\right]\left[\begin{array}{ll}
\mathrm{FR} & \dot{\alpha}_{\mathrm{I}}
\end{array}\right]+\left[\begin{array}{ll}
\mathrm{N}_{11} & \mathrm{~N}_{12} \\
\mathrm{~N}_{21} & \mathrm{~N}_{22}
\end{array}\right] \times\right. \\
& \left.\left.\left[\begin{array}{c}
\mathrm{FR}^{2} \\
\dot{\alpha_{\mathrm{I}}}
\end{array}\right]^{2}+\left[\begin{array}{c}
\mathrm{M}_{\mathrm{a}_{1}} \\
\mathrm{M}_{\mathrm{a}_{2}}
\end{array}\right]\right)\right)= \\
& \boldsymbol{S}^{\boldsymbol{T}}\left(\left[\begin{array}{ll}
\dot{\mathrm{M}}_{\mathrm{air}_{11}} & \dot{\mathrm{M}}_{\mathrm{air}_{12}} \\
\dot{\mathrm{M}}_{\mathrm{air}_{21}} & \dot{\mathrm{M}}_{\mathrm{air}_{22}}
\end{array}\right] \dot{\boldsymbol{S}}+\right. \\
& {\left[\begin{array}{l}
\mathrm{P}_{\text {motor }_{1}} \\
\mathrm{P}_{\text {motor }_{2}}
\end{array}\right]\left[\begin{array}{ll}
\dot{F R} & \dot{\alpha}_{\mathrm{I}}
\end{array}\right]+\left[\begin{array}{ll}
\mathrm{N}_{11} & \mathrm{~N}_{12} \\
\mathrm{~N}_{21} & \mathrm{~N}_{22}
\end{array}\right] \times\left[\begin{array}{c}
\dot{\mathrm{FR}} \\
\dot{\dot{\mathrm{I}}}
\end{array}\right]^{2}+} \\
& \left.\left[\begin{array}{l}
\mathrm{M}_{\mathrm{a}_{1}} \\
\mathrm{M}_{\mathrm{a}_{2}}
\end{array}\right]\right)
\end{aligned}
$$


suppose the control input is written as follows

$$
\begin{aligned}
& \widehat{U}=\widehat{U_{\text {Nonlınear }}}+\widehat{U_{\text {dis }}}= \\
& {\left[\begin{array}{ll}
\dot{M}_{\text {air }_{11}} & \dot{M}_{\text {air }_{12}} \\
\dot{M}_{\text {air }_{21}} & \dot{M}_{\text {air } 22}
\end{array}\right]^{-1}\left(\left[\begin{array}{l}
\mathrm{P}_{\text {motor }_{1}} \\
\mathrm{P}_{\text {motor }_{2}}
\end{array}\right]\left[\begin{array}{ll}
\mathrm{FR} & \dot{\alpha}_{\mathrm{I}}
\end{array}\right]+\right.} \\
& \left.\left[\begin{array}{ll}
\mathrm{N}_{11} & \mathrm{~N}_{12} \\
\mathrm{~N}_{21} & \mathrm{~N}_{22}
\end{array}\right] \times\left[\begin{array}{c}
\dot{\mathrm{FR}} \\
\dot{\dot{\alpha}_{\mathrm{I}}}
\end{array}\right]^{2}+\left[\begin{array}{l}
\mathrm{M}_{\mathrm{a}_{1}} \\
\mathrm{M}_{\mathrm{a}_{2}}
\end{array}\right]\right)+ \\
& \dot{\boldsymbol{S}}]\left[\begin{array}{ll}
\dot{\mathrm{M}}_{\mathrm{arr}_{11}} & \dot{\mathrm{M}}_{\mathrm{ar}_{12}} \\
\dot{\mathrm{M}}_{\mathrm{arr}_{21}} & \dot{\mathrm{M}}_{\mathrm{arr}_{22}}
\end{array}\right]+\boldsymbol{K} \cdot \boldsymbol{s} \boldsymbol{g n}(\boldsymbol{S})+ \\
& {\left[\begin{array}{l}
\mathrm{P}_{\text {motor }_{1}} \\
\mathrm{P}_{\text {motor }_{2}}
\end{array}\right]\left[\begin{array}{ll}
\dot{\mathrm{FR}} & \dot{\alpha}_{\mathrm{I}}
\end{array}\right]+\left[\begin{array}{ll}
\mathrm{N}_{11} & \mathrm{~N}_{12} \\
\mathrm{~N}_{21} & \mathrm{~N}_{22}
\end{array}\right] \times\left[\begin{array}{c}
\mathrm{FR} \\
\dot{\dot{\alpha}_{\mathrm{I}}}
\end{array}\right]^{2}+} \\
& {\left[\begin{array}{l}
\mathrm{M}_{\mathrm{a}_{1}} \\
\mathrm{M}_{\mathrm{a}_{2}}
\end{array}\right]}
\end{aligned}
$$

by replacing the equation (33) in (28)

$$
\begin{aligned}
& \dot{\boldsymbol{V}}=\boldsymbol{S}^{\boldsymbol{T}}\left(\left[\begin{array}{ll}
\dot{\mathrm{M}}_{\mathrm{air}_{11}} & \dot{\mathrm{M}}_{\mathrm{air}_{12}} \\
\dot{\mathrm{M}}_{\mathrm{air}_{21}} & \dot{\mathrm{M}}_{\mathrm{air}_{22}}
\end{array}\right] \dot{\boldsymbol{S}}+\right. \\
& {\left[\begin{array}{l}
\mathrm{P}_{\text {motor }_{1}} \\
\mathrm{P}_{\text {motor }_{2}}
\end{array}\right]\left[\begin{array}{ll}
\dot{\mathrm{FR}} & \dot{\alpha}_{\mathrm{I}}
\end{array}\right]+\left[\begin{array}{ll}
\mathrm{N}_{11} & \mathrm{~N}_{12} \\
\mathrm{~N}_{21} & \mathrm{~N}_{22}
\end{array}\right] \times\left[\begin{array}{c}
\mathrm{FR} \\
\dot{\alpha_{\mathrm{I}}}
\end{array}\right]^{2}+} \\
& {\left[\begin{array}{l}
\mathrm{M}_{\mathrm{a}_{1}} \\
\mathrm{M}_{\mathrm{a}_{2}}
\end{array}\right]-\left[\begin{array}{ll}
\dot{\mathrm{M}}_{\mathrm{arr}} & \dot{\mathrm{M}}_{\mathrm{arr}_{12}} \\
\dot{\mathrm{M}}_{\mathrm{arr}_{21}} & \dot{\mathrm{M}}_{\mathrm{arr}_{22}}
\end{array}\right] \dot{\boldsymbol{S}}-} \\
& {\left[\begin{array}{l}
\mathrm{P}_{\text {motor }_{1}} \\
\mathrm{P}_{\text {motor }_{2}}
\end{array}\right]\left[\begin{array}{ll}
\mathrm{FR} & \dot{\alpha_{\mathrm{I}}}
\end{array}\right]+\left[\begin{array}{ll}
\mathrm{N}_{11} & \mathrm{~N}_{12} \\
\mathrm{~N}_{21} & \mathrm{~N}_{22}
\end{array}\right] \times\left[\begin{array}{c}
\mathrm{FR}^{2} \\
\dot{\alpha_{\mathrm{I}}}
\end{array}\right]^{2} \boldsymbol{S}+} \\
& {\left[\begin{array}{l}
\mathrm{M}_{\mathrm{a}_{1}} \\
\mathrm{M}_{\mathrm{a}_{2}}
\end{array}\right]-\boldsymbol{K} \boldsymbol{s g n}(\boldsymbol{S})=} \\
& \boldsymbol{S}^{\boldsymbol{T}}\left(\left[\begin{array}{ll}
\dot{\mathrm{M}}_{\mathrm{arr}}-\dot{\mathrm{M}}_{11} & \dot{\mathrm{arr}}_{12} \\
\dot{\mathrm{M}}_{\mathrm{arr} 21} & \dot{\mathrm{M}}_{\mathrm{arr} 22}
\end{array}\right] \dot{\boldsymbol{S}}+\right. \\
& {\left[\begin{array}{l}
\mathrm{P}_{\text {motor }_{1}} \\
\mathrm{P}_{\text {motor }_{2}}
\end{array}\right]\left[\begin{array}{ll}
\mathrm{FR} & \dot{\alpha}_{\mathrm{I}}
\end{array}\right]+\left[\begin{array}{ll}
\mathrm{N}_{11} & \mathrm{~N}_{12} \\
\mathrm{~N}_{21} & \mathrm{~N}_{22}
\end{array}\right] \times\left[\begin{array}{c}
\dot{F R}^{2} \\
\dot{\dot{\alpha}_{\mathrm{I}}}
\end{array}\right]^{2} \boldsymbol{S}+} \\
& \left.\left[\begin{array}{l}
\mathrm{M}_{\mathrm{a}_{1}} \\
\mathrm{M}_{\mathrm{a}_{2}}
\end{array}\right]-\boldsymbol{K} \boldsymbol{s g n}(\boldsymbol{S})\right)
\end{aligned}
$$

and

$$
\begin{aligned}
& \mid\left[\begin{array}{ll}
\dot{\mathrm{M}}_{\text {arr }} & \dot{\mathrm{M}}_{\mathrm{arr}_{12}} \\
\dot{\mathrm{M}}_{\mathrm{arr}_{21}} & \dot{\mathrm{M}}_{\mathrm{arr}_{22}}
\end{array}\right] \dot{\boldsymbol{S}}+ \\
& {\left[\begin{array}{l}
\mathrm{P}_{\text {motor }_{1}} \\
\mathrm{P}_{\text {motor }_{2}}
\end{array}\right]\left[\begin{array}{ll}
\dot{\mathrm{FR}} & \dot{\alpha}_{\mathrm{I}}
\end{array}\right]+\left[\begin{array}{ll}
\mathrm{N}_{11} & \mathrm{~N}_{12} \\
\mathrm{~N}_{21} & \mathrm{~N}_{22}
\end{array}\right] \times\left[\begin{array}{c}
\dot{\mathrm{FR}} \\
\dot{\dot{\alpha}_{\mathrm{I}}}
\end{array}\right]^{2} \boldsymbol{S}+} \\
& {\left[\begin{array}{l}
\mathrm{M}_{\mathrm{a}_{1}} \\
\mathrm{M}_{\mathrm{a}_{2}}
\end{array}\right]|\leq|\left[\begin{array}{ll}
\dot{\mathrm{M}}_{\mathrm{arr}_{11}} & \dot{\mathrm{M}}_{\mathrm{arr}_{12}} \\
\dot{\mathrm{M}}_{\mathrm{arr}_{21}} & \dot{\mathrm{M}}_{\mathrm{arr}_{22}}
\end{array}\right] \dot{\boldsymbol{S}} \mid+} \\
& \mid\left[\begin{array}{l}
\mathrm{P}_{\text {motor }_{1}} \\
\mathrm{P}_{\text {motor }_{2}}
\end{array}\right]\left[\begin{array}{ll}
\mathrm{FR} & \dot{\alpha}_{\mathrm{I}}
\end{array}\right]+\left[\begin{array}{ll}
\mathrm{N}_{11} & \mathrm{~N}_{12} \\
\mathrm{~N}_{21} & \mathrm{~N}_{22}
\end{array}\right] \times\left[\begin{array}{c}
\dot{\mathrm{FR}} \\
\dot{\alpha_{\mathrm{I}}}
\end{array}\right]^{2} \boldsymbol{S}+ \\
& {\left[\begin{array}{l}
\mathrm{M}_{\mathrm{a}_{1}} \\
\mathrm{M}_{\mathrm{a}_{2}}
\end{array}\right]}
\end{aligned}
$$

The Lemma equation in robot arm system can be written as follows

$$
\begin{aligned}
& \boldsymbol{K}_{\boldsymbol{u}}=\left[\left|\left[\begin{array}{ll}
\dot{\mathrm{M}}_{\mathrm{arr}_{11}} & \dot{\mathrm{M}}_{\mathrm{arr}_{12}} \\
\dot{\mathrm{M}}_{\mathrm{arr}_{21}} & \dot{\mathrm{M}}_{\mathrm{arr}_{22}}
\end{array}\right] \dot{\boldsymbol{S}}\right|+\right. \\
& \mid\left[\begin{array}{l}
\mathrm{P}_{\text {motor }_{1}} \\
\mathrm{P}_{\text {motor }_{2}}
\end{array}\right]\left[\begin{array}{ll}
\dot{\mathrm{FR}} & \dot{\alpha}_{\mathrm{I}}
\end{array}\right]+\left[\begin{array}{ll}
\mathrm{N}_{11} & \mathrm{~N}_{12} \\
\mathrm{~N}_{21} & \mathrm{~N}_{22}
\end{array}\right] \times \\
& \left.\left[\begin{array}{c}
\mathrm{FR}^{2} \\
\dot{\alpha_{\mathrm{I}}}
\end{array}\right]^{2} \boldsymbol{S}+\left[\begin{array}{l}
\mathrm{M}_{\mathrm{a}_{1}} \\
\mathrm{M}_{\mathrm{a}_{2}}
\end{array}\right] \mid+\boldsymbol{\eta}\right]_{\boldsymbol{i}}, \boldsymbol{i}=\mathbf{1}, \mathbf{2 , 3}, \mathbf{4}, \ldots
\end{aligned}
$$

and finally;

$$
\dot{V} \leq-\sum_{i=1}^{n} \eta_{i}\left|S_{i}\right|
$$

\subsection{Fuzzy Logic Methodology}

Based on foundation of fuzzy logic methodology; fuzzy logic management has played important rule to design nonlinear management for nonlinear and uncertain systems [16]. However the application area for fuzzy control is really wide, the basic form for all command types of controllers consists of;

Input fuzzification (binary-to-fuzzy $[\mathrm{B} / \mathrm{F}]$ conversion)

Fuzzy rule base (knowledge base), Inference engine and Output defuzzification (fuzzy-to-binary [F/B] conversion). Figure 1 shows the fuzzy controller part.

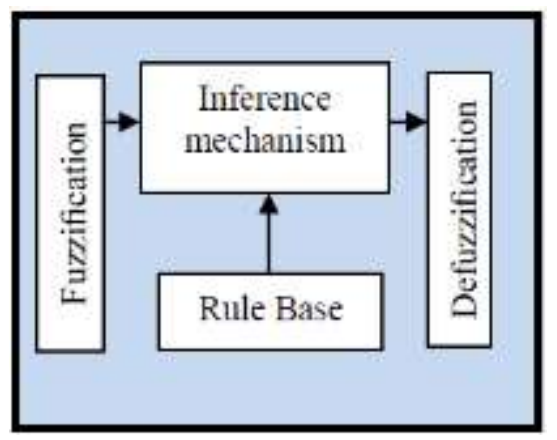

Fig. 1: Fuzzy Controller Part

The fuzzy inference engine offers a mechanism for transferring the rule base in fuzzy set which it is divided into two most important methods, namely, Mamdani method and Sugeno method. Mamdani method is one of the common fuzzy inference systems and he designed one of the first fuzzy managements to control of system engine. Mamdani's fuzzy inference system is divided into four major steps: fuzzification, rule evaluation, aggregation of the rule outputs and defuzzification. Michio Sugeno use a singleton as a membership function of the rule consequent part. The following definition shows the Mamdani and Sugeno fuzzy rule base [22-33] 


\section{if $x$ is $A$ and $y$ is $B$ then $z$ is $C$ 'mamdani' if $x$ is $A$ and $y$ is B then $z$ is $f(x, y)$ 'sugeno'}

When $x$ and $y$ have crisp values fuzzification calculates the membership degrees for antecedent part. Rule evaluation focuses on fuzzy operation $(A N D / O R)$ in the antecedent of the fuzzy rules. The aggregation is used to calculate the output fuzzy set and several methodologies can be used in fuzzy logic controller aggregation, namely, Max-Min aggregation, Sum-Min aggregation, Max-bounded product, Max-drastic product, Max-bounded sum, Max-algebraic sum and Min-max. Defuzzification is the last step in the fuzzy inference system which it is used to transform fuzzy set to crisp set. Consequently defuzzification's input is the aggregate output and the defuzzification's output is a crisp number. Centre of gravity method ( $C O G)$ and Centre of area method $(C O A)$ are two most common defuzzification methods.

\section{Methodology:}

Based on the dynamic formulation of IC engine, (3), and the industrial PD law (4) in this paper we discuss about regulation problem, the desired position is constant, i.e., $\dot{q}_{d}=0$. In most IC engine control, desired joint positions are generated by the trajectory planning. The objective of robot control is to design the input torque in (1) such that the tracking error

$$
e=F R_{d}-F R I_{a}
$$

When the dynamic parameters of robot formulation known, the PD control formulation (10) shoud include a compensator as

$$
P F I=-k_{p} e-k_{d} e+(G+F)
$$

Where $G$ is gravity and $F$ is appositive definite diagonal matrix friction term (coulomb friction). If we use a Lyapunov function candidate as

$$
\begin{gathered}
V_{p d}=\frac{1}{2} \dot{F R} R^{T}\left[\begin{array}{ll}
\dot{\mathrm{M}}_{\mathrm{air}_{11}} & \dot{\mathrm{M}}_{\text {air }_{12}} \\
\dot{\mathrm{M}}_{\text {air }_{21}} & \dot{\mathrm{M}}_{\text {air }_{22}}
\end{array}\right] \dot{F R} \\
+\frac{1}{2} e^{T} k_{p} e \\
\dot{V}_{p d}=-\dot{F R^{T}} k_{d} \dot{F R} \leq 0
\end{gathered}
$$

It is easy to known $F R=0$ and $e=0$ are only initial conditions in $\Omega=\{[\dot{q}, e]: \dot{V}=0\}$, for which $[\dot{F R}, e] \in$ $\Omega$ for al $1 t \leq 0$. By the LaSalle's invariance principle, $e \rightarrow 0$ and $\dot{e} \rightarrow 0$. When $\mathrm{G}$ and $\mathrm{F}$ in (10) are unknown, a fuzzy logic can be used to approximate them as

$$
f(x)=\sum_{l=1}^{M} \theta^{l} \mathcal{E}^{l}(x)=\theta^{T} \mathcal{E}(x)
$$

Where $\quad \theta=\left(\theta^{1}, \ldots, \theta^{M}\right)^{T}, \mathcal{E}(x)=$ $\left(\mathcal{E}^{1}(x), \ldots, \mathcal{E}^{M}(x)\right)^{T}$, and $\mathcal{E}^{l}(x)=$ $: \prod_{i=1}^{n} \frac{{ }_{A_{i}^{l^{l}}}\left(x_{i}\right)}{} \sum_{l=1}^{M}\left(\prod_{i=1}^{n} \mu_{A_{i}^{l}}\left(x_{i}\right)\right) . \quad \theta^{1}, \ldots, \theta^{M} \quad$ are adjustable parameters in (43). $\mu_{A_{1}^{1}}\left(x_{1}\right), \ldots, \mu_{A_{n}^{m}}\left(x_{n}\right)$ are given membership functions whose parameters will not change over time.

The second type of fuzzy systems is given by

$$
f(x)=\frac{\sum_{l=1}^{M} \theta^{l}\left[\prod_{i=1}^{n} \exp \left(-\left(\frac{x_{i}-\alpha_{i}^{l}}{\delta_{i}^{l}}\right)^{2}\right)\right]}{\sum_{l=1}^{M}\left[\prod_{i=1}^{n} \exp \left(-\left(\frac{x_{i}-\alpha_{i}^{l}}{\delta_{i}^{l}}\right)^{2}\right)\right]}
$$

Where $\theta^{l}, \alpha_{i}^{l}$ and $\delta_{i}^{l}$ are all adjustable parameters. From the universal approximation theorem, we know that we can find a fuzzy system to estimate any continuous function. For the first type of fuzzy systems, we can only adjust $\theta^{l}$ in (44). We define $f^{\wedge}(x \mid \theta)$ as the approximator of the real function $f(x)$.

$$
f^{\wedge}(x \mid \theta)=\theta^{T} \varepsilon(x)
$$

We define $\theta^{*}$ as the values for the minimum error:

$$
\theta^{*}=\arg \min _{\theta \in \Omega}\left[\sup _{x \in U}\left|f^{\wedge}(x \mid \theta)-g(x)\right|\right]
$$

Where $\Omega$ is a constraint set for $\theta$. For specific $x, \sup _{x \in U}\left|f^{\wedge}\left(x \mid \theta^{*}\right)-f(x)\right| \quad$ is the minimum approximation error we can get.

We used the first type of fuzzy systems (43) to estimate the nonlinear system (11) the fuzzy formulation can be write as below;

$$
\begin{aligned}
f(x \mid \theta) & =\theta^{T} \varepsilon(x) \\
& =\frac{\sum_{l=1}^{n} \theta^{l}\left[\mu_{A^{l}}(x)\right]}{\sum_{l=1}^{n}\left[\mu_{A^{l}}(x)\right]}
\end{aligned}
$$

Where $\theta^{1}, \ldots, \theta^{n}$ are adjusted by an adaptation law. The adaptation law is designed to minimize the parameter errors of $\theta-\theta^{*}$. The SISO fuzzy system is define as

$$
f(x)=\ominus^{T} \varepsilon(x)
$$

Where

$$
\ominus^{T}=\left(\theta_{1}, \ldots, \theta_{m}\right)^{T}=\left[\begin{array}{c}
\theta_{1}^{1}, \theta_{1}^{2}, \ldots, \theta_{1}^{M} \\
\theta_{2}^{1}, \theta_{2}^{2}, \ldots, \theta_{2}^{M} \\
\vdots \\
\theta_{m}^{1}, \theta_{m}^{2}, \ldots, \theta_{m}^{M}
\end{array}\right]
$$


$\varepsilon(x)=\left(\varepsilon^{1}(x), \ldots, \varepsilon^{M}(x)\right)^{T}, \quad \varepsilon^{1}(x)=\prod_{i=1}^{n} \mu_{A_{i}^{l}}\left(x_{i}\right) /$

$\sum_{l=1}^{M}\left(\prod_{i=1}^{n} \mu_{A_{i}^{l}}\left(x_{i}\right)\right)$, and $\mu_{A_{i}^{l}}\left(x_{i}\right)$ is defined in (47). To reduce the number of fuzzy rules, we divide the fuzzy system in to three parts:

$$
\begin{aligned}
& F^{1}(q, \dot{q})=\ominus^{1^{T}} \varepsilon(F R, \dot{F R}) \\
& =\left[\theta_{1}^{1^{T}} \varepsilon(F R, \dot{F R}), \ldots, \theta_{m}^{1^{T}} \varepsilon(F R, \dot{F R})\right]^{T} \\
& F^{2}(F R, \ddot{F R} r)=\ominus^{2^{T}} \varepsilon(F R, \ddot{F R}) \\
& =\left[\theta_{1}^{2^{T}} \varepsilon\left(F R, \ddot{F R}{ }_{r}\right), \ldots, \theta_{m}^{2^{T}} \varepsilon\left(F R, \ddot{F R} R_{r}\right)\right]^{T} \\
& F^{3}(F R, \ddot{F R})=\ominus^{3^{T}} \varepsilon(F R, \ddot{F R}) \\
& =\left[\theta_{1}^{3^{T}} \varepsilon(F R, \ddot{F R}), \ldots, \theta_{m}^{3^{T}} \varepsilon(F R, \ddot{F R})\right]^{T}
\end{aligned}
$$

The control security input is given by

$$
\begin{aligned}
& \tau=\left[\begin{array}{ll}
\dot{\mathrm{M}}_{\text {air }_{11}} & \dot{\mathrm{M}}_{\text {air }_{12}} \\
\dot{\mathrm{M}}_{\mathrm{air}_{21}} & \dot{\mathrm{M}}_{\mathrm{air}_{22}}
\end{array}\right] F \ddot{R}_{r}+ \\
& {\left[\begin{array}{ll}
\mathrm{P}_{\text {motor }_{1}} \\
\mathrm{P}_{\text {motor }_{2}}
\end{array}\right]\left[\begin{array}{ll}
\mathrm{FR} & \dot{\alpha}_{\mathrm{I}}
\end{array}\right]+\left[\begin{array}{ll}
\mathrm{N}_{11} & \mathrm{~N}_{12} \\
\mathrm{~N}_{21} & \mathrm{~N}_{22}
\end{array}\right] \times} \\
& {\left[\begin{array}{c}
\mathrm{FR}^{2} \\
\dot{\alpha}_{\mathrm{I}}
\end{array}\right]^{2}+\left[\begin{array}{l}
\mathrm{M}_{\mathrm{a}_{1}} \\
\mathrm{M}_{\mathrm{a}_{2}}
\end{array}\right]+F^{1}(F R, \dot{F R})+} \\
& F^{2}\left(F R, \ddot{F R} R_{r}\right)+F^{3}(F R, \ddot{F R})-K_{p} e-K_{v} \dot{e}
\end{aligned}
$$

Where $\left[\begin{array}{ll}\dot{\mathrm{M}}_{\text {air }_{11}} & \dot{\mathrm{M}}_{\mathrm{air}_{12}} \\ \dot{\mathrm{M}}_{\text {air }_{21}} & \dot{\mathrm{M}}_{\mathrm{air}_{22}}\end{array}\right]^{\wedge},\left[\begin{array}{l}\mathrm{P}_{\text {motor }_{1}} \\ \text { Potor }_{2}\end{array}\right]\left[\begin{array}{ll}\text { FR } & \dot{\alpha}_{\mathrm{I}}\end{array}\right]+$ $\left[\begin{array}{ll}\mathrm{N}_{11} & \mathrm{~N}_{12} \\ \mathrm{~N}_{21} & \mathrm{~N}_{22}\end{array}\right] \times\left[\begin{array}{c}\mathrm{FR} \\ \dot{\alpha_{I}}\end{array}\right]^{2}+\left[\begin{array}{c}\mathrm{M}_{\mathrm{a}_{1}} \\ \mathrm{M}_{\mathrm{a}_{2}}\end{array}\right]$ are the estimations of $\left[\begin{array}{ll}\dot{\mathrm{M}}_{\text {air }_{11}} & \dot{\mathrm{M}}_{\text {air }} \\ \dot{\mathrm{M}}_{\text {air }} & \dot{\mathrm{M}}_{\text {air }}\end{array}\right]$.

Based on sliding mode formulation (27) and PD linear methodology (4);

$$
S_{N e w}=(\dot{e}+\lambda e)
$$

And $U_{\text {switch }}$ is obtained by

$$
\begin{aligned}
& \mathrm{U}_{\text {switch }}=\mathrm{K}(\overrightarrow{\mathrm{x}}, \mathrm{t}) \cdot \operatorname{sgn}\left(\mathrm{S}_{\mathrm{New}}\right)=\mathrm{K}(\overrightarrow{\mathrm{x}}, \mathrm{t}) \cdot \\
& \operatorname{sgn}(K(\dot{e}+\lambda e))
\end{aligned}
$$

The Lyapunov function in this design is defined as

$$
V=\frac{1}{2} S^{T} M S+\frac{1}{2} \sum_{J=1}^{M} \frac{1}{\gamma_{s j}} \phi^{T} \cdot \phi_{j}
$$

where $\gamma_{s j}$ is a positive coefficient, $\boldsymbol{\phi}=\boldsymbol{\theta}^{*}-\boldsymbol{\theta}, \boldsymbol{\theta}^{*}$ is minimum error and $\theta$ is adjustable parameter. Since $\dot{M}-2 V$ is skew-symetric matrix;

$$
\begin{aligned}
& S^{T}\left[\begin{array}{ll}
\dot{\mathrm{M}}_{\mathrm{air}_{11}} & \dot{\mathrm{M}}_{\mathrm{air}_{12}} \\
\dot{\mathrm{M}}_{\mathrm{air}_{21}} & \dot{\mathrm{M}}_{\mathrm{air}_{22}}
\end{array}\right] \dot{S}+ \\
& \frac{1}{2} S^{T}\left[\begin{array}{ll}
\dot{\mathrm{M}}_{\mathrm{arr}_{11}} & \dot{\mathrm{M}}_{\mathrm{arr}_{12}} \\
\dot{\mathrm{M}}_{\text {arr }} & \dot{\mathrm{M}}_{\mathrm{arr}_{22}}
\end{array}\right] S= \\
& S^{T}\left(\left[\begin{array}{ll}
\dot{\mathrm{M}}_{\mathrm{air}_{11}} & \dot{\mathrm{M}}_{\mathrm{air}_{12}} \\
\dot{\mathrm{M}}_{\mathrm{air}_{21}} & \dot{\mathrm{M}}_{\mathrm{air}_{22}}
\end{array}\right] \dot{S}+V S\right)
\end{aligned}
$$

If the dynamic formulation of IC engine defined by

$$
\begin{aligned}
& \tau=\left[\begin{array}{ll}
\dot{\mathrm{M}}_{\text {air }_{11}} & \dot{\mathrm{M}}_{\text {air }_{12}} \\
\dot{\mathrm{M}}_{\mathrm{air}_{21}} & \dot{\mathrm{M}}_{\text {air }}
\end{array}\right] \ddot{F R}+ \\
& {\left[\begin{array}{l}
\mathrm{P}_{\text {motor }_{1}} \\
\mathrm{P}_{\text {motor }_{2}}
\end{array}\right]\left[\begin{array}{ll}
\mathrm{FR} & \dot{\alpha}_{\mathrm{I}}
\end{array}\right]+\left[\begin{array}{ll}
\mathrm{N}_{11} & \mathrm{~N}_{12} \\
\mathrm{~N}_{21} & \mathrm{~N}_{22}
\end{array}\right] \times\left[\begin{array}{c}
\mathrm{FR}^{2} \\
\dot{\mathrm{d}}_{\mathrm{I}}
\end{array}\right]^{2}+} \\
& {\left[\begin{array}{l}
\mathrm{M}_{\mathrm{a}_{1}} \\
\mathrm{M}_{\mathrm{a}_{2}}
\end{array}\right]}
\end{aligned}
$$

the controller formulation is defined by

$$
\begin{aligned}
& \tau=\left[\begin{array}{ll}
\dot{\mathrm{M}}_{\mathrm{arr}_{11}} & \dot{\mathrm{M}}_{\mathrm{arr}_{12}} \\
\dot{\mathrm{M}}_{\mathrm{arr}_{21}} & \dot{\mathrm{M}}_{\mathrm{arr}_{22}}
\end{array}\right] \ddot{F R_{r}}+ \\
& {\left[\begin{array}{l}
\mathrm{P}_{\text {motor }_{1}} \\
\mathrm{P}_{\text {motor }_{2}}
\end{array}\right]\left[\begin{array}{ll}
\mathrm{FR} & \dot{\mathrm{\alpha}}_{\mathrm{I}}
\end{array}\right]+\left[\begin{array}{ll}
\mathrm{N}_{11} & \mathrm{~N}_{12} \\
\mathrm{~N}_{21} & \mathrm{~N}_{22}
\end{array}\right] \times\left[\begin{array}{c}
\dot{\mathrm{FR}} \\
\dot{\dot{\alpha}_{\mathrm{I}}}
\end{array}\right]^{2} \dot{F R_{r}}} \\
& {\left[\begin{array}{l}
\mathrm{M}_{\mathrm{a}_{1}} \\
\mathrm{M}_{\mathrm{a}_{2}}
\end{array}\right]-\lambda S-K}
\end{aligned}
$$

According to (57) and (58)

$$
\begin{aligned}
& {\left[\begin{array}{ll}
\dot{\mathrm{M}}_{\text {air }_{11}} & \dot{\mathrm{M}}_{\text {air }_{12}} \\
\dot{\mathrm{M}}_{\mathrm{air}_{21}} & \dot{\mathrm{M}}_{\text {air }_{22}}
\end{array}\right] \ddot{F R}+\left[\begin{array}{l}
\mathrm{P}_{\text {motor }_{1}} \\
\mathrm{P}_{\text {motor }_{2}}
\end{array}\right]\left[\begin{array}{ll}
\dot{\mathrm{FR}} & \dot{\alpha}_{\mathrm{I}}
\end{array}\right]+} \\
& {\left[\begin{array}{ll}
\mathrm{N}_{11} & \mathrm{~N}_{12} \\
\mathrm{~N}_{21} & \mathrm{~N}_{22}
\end{array}\right] \times\left[\begin{array}{c}
\dot{F R} \\
\dot{\alpha_{I}}
\end{array}\right]^{2}+\left[\begin{array}{c}
\mathrm{M}_{\mathrm{a}_{1}} \\
\mathrm{M}_{\mathrm{a}_{2}}
\end{array}\right]=} \\
& {\left[\begin{array}{ll}
\dot{\mathrm{M}}_{\mathrm{arr}_{11}} & \dot{\mathrm{M}}_{\mathrm{arr}_{12}} \\
\dot{\mathrm{M}}_{\mathrm{arr}_{21}} & \dot{\mathrm{M}}_{\mathrm{arr}_{22}}
\end{array}\right] \ddot{F R}+} \\
& {\left[\begin{array}{l}
\mathrm{P}_{\text {motor }_{1}} \\
\mathrm{P}_{\text {motor }_{2}}
\end{array}\right]\left[\begin{array}{ll}
\mathrm{FR} & \dot{\alpha}_{\mathrm{I}}
\end{array}\right]+\left[\begin{array}{ll}
\mathrm{N}_{11} & \mathrm{~N}_{12} \\
\mathrm{~N}_{21} & \mathrm{~N}_{22}
\end{array}\right] \times\left[\begin{array}{c}
\dot{\mathrm{FR}} \\
\dot{\dot{\alpha}_{\mathrm{I}}}
\end{array}\right]^{2}+} \\
& {\left[\begin{array}{l}
\mathrm{M}_{\mathrm{a}_{1}} \\
\mathrm{M}_{\mathrm{a}_{2}}
\end{array}\right]-\lambda S-K}
\end{aligned}
$$

Since $\ddot{F} \boldsymbol{R}_{r}=\ddot{F R}-\boldsymbol{S}$ and $\ddot{F} \boldsymbol{R}_{r}=\ddot{F R}-\dot{\boldsymbol{S}}$

$$
\begin{aligned}
& {\left[\begin{array}{ll}
\dot{\mathrm{M}}_{\text {air }_{11}} & \dot{\mathrm{M}}_{\text {air }} 12 \\
\dot{\mathrm{M}}_{\text {air }} & \dot{\mathrm{M}}_{\text {air }_{22}}
\end{array}\right] \dot{S}+\left(\left[\begin{array}{l}
\mathrm{P}_{\text {motor }_{1}} \\
\mathrm{P}_{\text {motor }_{2}}
\end{array}\right]\left[\begin{array}{ll}
\dot{\mathrm{FR}} & \dot{\alpha}_{\mathrm{I}}
\end{array}\right]+\right.} \\
& \left.\left[\begin{array}{ll}
\mathrm{N}_{11} & \mathrm{~N}_{12} \\
\mathrm{~N}_{21} & \mathrm{~N}_{22}
\end{array}\right] \times\left[\begin{array}{c}
\mathrm{FR}^{2} \\
\dot{\alpha}_{\mathrm{I}}
\end{array}\right]^{2}+\lambda\right) S=\Delta f-K
\end{aligned}
$$




$$
\begin{aligned}
{\left[\begin{array}{ll}
\dot{\mathrm{M}}_{\text {air } 11} & \dot{\mathrm{M}}_{\mathrm{air}_{12}} \\
\dot{\mathrm{M}}_{\mathrm{air}_{21}} & \dot{\mathrm{M}}_{\mathrm{air}_{22}}
\end{array}\right] \dot{\boldsymbol{S}} } \\
=\boldsymbol{\Delta} \boldsymbol{f}-\boldsymbol{K}-\left[\begin{array}{l}
\mathrm{P}_{\text {motor }_{1}} \\
\mathrm{P}_{\text {motor }_{2}}
\end{array}\right]\left[\begin{array}{ll}
\mathrm{FR} & \dot{\alpha}_{\mathrm{I}}
\end{array}\right] \\
+\left[\begin{array}{ll}
\mathrm{N}_{11} & \mathrm{~N}_{12} \\
\mathrm{~N}_{21} & \mathrm{~N}_{22}
\end{array}\right] \times\left[\begin{array}{c}
\dot{\mathrm{FR}} \\
\dot{\alpha_{\mathrm{I}}}
\end{array}\right]^{2} \boldsymbol{S}-\boldsymbol{\lambda} \boldsymbol{S}
\end{aligned}
$$

This method has two main management's coefficients, $K_{p}$ and $K_{V}$. To tune and optimize these parameters mathematical formulation is used

$$
\begin{aligned}
& U=U_{f u z z y}+U_{\text {sliding }}+U_{P D} \\
& \mathrm{U}=\mathrm{U}_{\text {fuzzy }}+\mathrm{U}_{\text {switch }}= \\
& {\left[\begin{array}{ll}
\dot{\mathrm{M}}_{\text {air }_{11}} & \dot{\mathrm{M}}_{\text {air }_{12}} \\
\dot{\mathrm{M}}_{\text {air }_{21}} & \dot{\mathrm{M}}_{\text {air }_{22}}
\end{array}\right]^{-1}\left(\left[\begin{array}{l}
\mathrm{P}_{\text {motor }_{1}} \\
\mathrm{P}_{\text {motor }_{2}}
\end{array}\right]\left[\begin{array}{ll}
\dot{\mathrm{FR}} & \dot{\alpha}_{\mathrm{I}}
\end{array}\right]+\right.} \\
& \left.\left[\begin{array}{ll}
\mathrm{N}_{11} & \mathrm{~N}_{12} \\
\mathrm{~N}_{21} & \mathrm{~N}_{22}
\end{array}\right] \times\left[\begin{array}{c}
\mathrm{FR} \\
\dot{\dot{\alpha}_{\mathrm{I}}}
\end{array}\right]^{2}\right)+ \\
& \dot{\boldsymbol{S}}]\left[\begin{array}{ll}
\dot{\mathrm{M}}_{\mathrm{air}_{11}} & \dot{\mathrm{M}}_{\mathrm{air}_{12}} \\
\dot{\mathrm{M}}_{\mathrm{air}_{21}} & \dot{\mathrm{M}}_{\mathrm{air}_{22}}
\end{array}\right]+\boldsymbol{K} \cdot \operatorname{sgn}(\boldsymbol{S})+ \\
& \frac{\sum_{\mathrm{l}=1}^{\mathrm{M}} \theta^{\mathrm{l}}\left[\Pi_{\mathrm{i}=1}^{\mathrm{n}} \exp \left(-\left(\frac{\mathrm{x}_{\mathrm{i}}-\alpha_{\mathrm{i}}^{\mathrm{l}}}{\delta_{\mathrm{i}}^{\mathrm{l}}}\right)^{2}\right)\right]}{\sum_{\mathrm{l}=1}^{\mathrm{M}}\left[\Pi_{\mathrm{i}=1}^{\mathrm{n}} \exp \left(-\left(\frac{\mathrm{x}_{\mathrm{i}}-\alpha_{\mathrm{i}}^{\mathrm{l}}}{\delta_{\mathrm{i}}^{\mathrm{l}}}\right)^{2}\right)\right]}+\boldsymbol{K}_{\boldsymbol{p}_{\boldsymbol{a}}} \boldsymbol{e}_{\mathbf{1}}+ \\
& K_{V_{a}} \dot{e}_{1}+K_{I_{a}} \sum e_{1}
\end{aligned}
$$

The most important different between PD+SMC and $\mathrm{PD}+\mathrm{SMC}+\mathrm{FL}$ is the uncertainty. In $\mathrm{PD}+\mathrm{SMC}$ the uncertainty is $d=G+F+f$. The sliding mode gain must be bigger than its upper bound. It is not an easy job because this term includes tracking errors $e_{1}$ and $\dot{q}_{1}$. While in PD+SMC+FL, the uncertainty $\eta$ is the fuzzy approximation error for $G+F+f$.

$$
G+F+f=\frac{\sum_{l=1}^{M} \theta^{l}\left[\prod_{i=1}^{n} \exp \left(-\left(\frac{x_{i}-\alpha_{i}^{l}}{\delta_{i}^{l}}\right)^{2}\right)\right]}{\sum_{l=1}^{M}\left[\prod_{i=1}^{n} \exp \left(-\left(\frac{x_{i}-\alpha_{i}^{l}}{\delta_{i}^{l}}\right)^{2}\right)\right]}
$$

It is usually is smaller than $G+F+f$; and the upper bound of it is easy to be estimated.

\section{Results}

In this section, we use a benchmark model, IC engine, to evaluate our control algorithms [22]. We compare the following managements: classical PD management, PD fuzzy management and serial fuzzy sliding mode PD management which is proposed in this paper. The simulation was implemented in MATLAB/SIMULINK environment.

Close loop response of IC engine fuel ratio: Figure 2 illustrates the tracking performance in three types of management; linear PD management, linear PD management based on fuzzy logic estimator and nonlinear estimator based on fuzzy logic and sliding mode management.
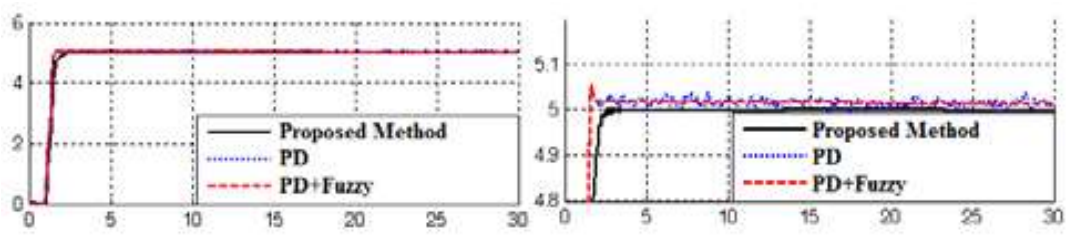

Fig. 2: Linear PD, PD+FLC and Proposed method fuel ratio following without disturbance

Based on Figure 2; pure PD management has oscillation in first and three links, because IC engine is a highly nonlinear management and control of this system by linear method is very difficult. Based on above graph, however PD+FUZZY controller is a nonlinear methodology but it has difficulty to control this plant because it is a model base controller.
Close loop response of fuel ratio following in presence of load disturbance: Figure 3 demonstrates the power disturbance elimination in three types of controller in presence of disturbance for IC engine. The disturbance rejection is used to test the robustness comparisons of these three methodologies.
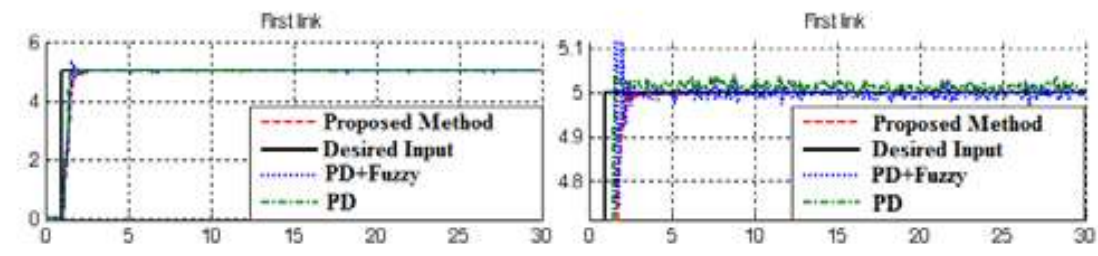

Fig 3: Linear PD, PD+FLC and Proposed method fuel ratio following with disturbance 
Based on Figure 3; by comparison with the PD and PD+FLC, proposed serial compensator PD+Fuzzy+SMC is more stable and robust and our method doesn't have any chattering and oscillation.

\section{Conclusion}

The main contributions of the paper are twofold. The structure of fuzzy PD control with sliding mode compensation is new. We propose parallel structure and chattering free compensator: parallel compensation, and chattering free method. The key technique is dead-zone, such that fuzzy control and sliding mode control can be switched automatically. The stability analysis of fuzzy sliding mode PD manages is also new. Stability analysis of fuzzy PD algorithm with first-order or second-order sliding mode is not published in the literature. The benefits of the proposed method; the chattering effects of fuzzy sliding mode PD methodology, the slow convergence of the fuzzy $\mathrm{PD}$ and the chattering problem of sliding mode PD method are avoided effectively.

\section{Acknowledgment}

The authors would like to thank the anonymous reviewers for their careful reading of this paper and for their helpful comments. This work was supported by the SSP Research and Development Corporation Program of Iran under grant no. 2012-Persian Gulf-2B.

\section{References}

[1] Heywood, J., "Internal Combustion Engine Fundamentals”, McGraw-Hill, New York, 1988.

[2] J. G. Rivard, "Closed-loop Electronic Fuel Injection Control of the IC Engine," in Society of Automotive Engineers, 1973.

[3] J. F. Cassidy, et al, "On the Design of Electronic Automotive Engine Controls using linear Quadratic Control Theory," IEEE Trans on Control Systems, vol. AC-25, October 1980.

[4] W. E. Powers, "Applications of Optimal Control and Kalman Filtering to Automotive Systems," International Journal of Vehicle Design, vol. Applications of Control Theory in the Automotive Industry, 1983.

[5] N. F. Benninger, et al, "Requirements and Perfomance of Engine Management Systems under Transient Conditions," in Society of Automotive Engineers, 1991.

[6] C. H. Onder, et al, "Model-Based Multivariable Speed and Air-to-Fuel Ratio Control of an SI Engine," in Society of Automotive Engineers, 1993.
[7] S. B. Cho, et al, "An Observer-based Controller Design Method for Automotive Fuel-Injection Systems," in American Controls Conference, 1993, pp. 2567-2571.

[8] T. Kume, et al, "Combustion Technologies for Direct Injection SI Engine," in Society of Automotive Engineers, 1996.

[9] M. Bazregar, Farzin Piltan, A. Nabaee and M.M. Ebrahimi, "Parallel Soft Computing Control Optimization Algorithm for Uncertainty Dynamic Systems", International Journal of Advanced Science and Technology, 51, 2013.

[10] Farzin Piltan, M.H. Yarmahmoudi, M. Mirzaei, S. Emamzadeh, Z. Hivand, "Design Novel Fuzzy Robust Feedback Linearization Control with Application to Robot Manipulator", International Journal of Intelligent Systems and Applications, 5(5), 2013

[11] Sh. Tayebi Haghighi, S. Soltani, Farzin Piltan, M. kamgari, S. Zare, "Evaluation Performance of IC Engine: Linear Tunable Gain Computed Torque Controller Vs. Sliding Mode Controller", International Journal of Intelligent Systems and Applications, 5(6), 2013.

[12] Farzin Piltan, A. R. Salehi \& Nasri B Sulaiman,"Design Artificial Robust Control of Second Order System Based on Adaptive Fuzzy Gain Scheduling", World Applied Science Journal (WASJ), 13 (5): 1085-1092, 2011.

[13] Farzin Piltan, N. Sulaiman, Atefeh Gavahian, Samira Soltani \& Samaneh Roosta, "Design Mathematical Tunable Gain PID-Like Sliding Mode Fuzzy Controller with Minimum Rule Base", International Journal of Robotic and Automation, 2 (3): 146-156, 2011.

[14] Farzin Piltan , N. Sulaiman, Zahra Tajpaykar, Payman Ferdosali \& Mehdi Rashidi, "Design Artificial Nonlinear Robust Controller Based on CTLC and FSMC with Tunable Gain", International Journal of Robotic and Automation, 2 (3): 205-220, 2011.

[15] Farzin Piltan, Mohammad Mansoorzadeh, Saeed Zare, Fatemeh Shahriarzadeh, Mehdi Akbari, "Artificial tune of fuel ratio: Design a novel siso fuzzy backstepping adaptive variable structure control", International Journal of Electrical and Computer Engineering (IJECE), 3 (2): 183-204, 2013.

[16] Farzin Piltan, M. Bazregar, M. Kamgari, M. Akbari, M. Piran, "Adjust the fuel ratio by high impact chattering free sliding methodology with application to automotive engine", International Journal of Hybrid Information Technology (IJHIT), 6 (1): 13-24, 2013. 
[17] Shahnaz Tayebi Haghighi, S. Soltani, Farzin Piltan, M. Kamgari, S. Zare, "Evaluation Performance of IC Engine: linear tunable gain computed torque controller Vs. Sliding mode controller", I. J. Intelligent system and application, 6 (6): 78-88, 2013.

[18] Farzin Piltan, N. Sulaiman, Payman Ferdosali \& Iraj Assadi Talooki, "Design Model Free Fuzzy Sliding Mode Control: Applied to Internal Combustion Engine", International Journal of Engineering, 5 (4):302-312, 2011.

[19] Farzin Piltan, N. Sulaiman, A. Jalali \& F. Danesh Narouei, "Design of Model Free Adaptive Fuzzy Computed Torque Controller: Applied to Nonlinear Second Order System", International Journal of Robotics and Automation, 2 (4):245-257, 2011

[20] A. Jalali, Farzin Piltan, M. Keshtgar, M. Jalali, "Colonial Competitive Optimization Sliding Mode Controller with Application to Robot Manipulator", International Journal of Intelligent Systems and Applications, 5(7), 2013.

[21] Farzin Piltan, Amin Jalali, N. Sulaiman, Atefeh Gavahian \& Sobhan Siamak, "Novel Artificial Control of Nonlinear Uncertain System: Design a Novel Modified PSO SISO Lyapunov Based Fuzzy Sliding Mode Algorithm”, International Journal of Robotics and Automation, 2 (5): 298316,2011 .

[22] Farzin Piltan, N. Sulaiman, Iraj Asadi Talooki \& Payman Ferdosali, "Control of IC Engine: Design a Novel MIMO Fuzzy Backstepping Adaptive Based Fuzzy Estimator Variable Structure Control", International Journal of Robotics and Automation, 2 (5):360-380, 2011.

[23] Farzin Piltan, N. Sulaiman, S.Soltani, M. H. Marhaban \& R. Ramli, "An Adaptive Sliding Surface Slope Adjustment in PD Sliding Mode Fuzzy Control For Robot Manipulator", International Journal of Control and Automation, 4 (3): 65-76, 2011.

[24] Farzin Piltan, N. Sulaiman, Mehdi Rashidi, Zahra Tajpaikar \& Payman Ferdosali, "Design and Implementation of Sliding Mode Algorithm: Applied to Robot Manipulator-A Review", International Journal of Robotics and Automation, 2 (5):265-282, 2011.

[25] Farzin Piltan, N. Sulaiman , Arash Zargari, Mohammad Keshavarz \& Ali Badri, "Design PIDLike Fuzzy Controller with Minimum Rule Base and Mathematical Proposed On-line Tunable Gain: Applied to Robot Manipulator", International Journal of Artificial Intelligence and Expert System, 2 (4):184-195, 2011.

[26] Farzin Piltan, SH. Tayebi HAGHIGHI, N. Sulaiman, Iman Nazari \& Sobhan Siamak,
"Artificial Control of PUMA Robot Manipulator: A-Review of Fuzzy Inference Engine and Application to Classical Controller", International Journal of Robotics and Automation, 2 (5):401-425, 2011.

[27] A. Salehi, Farzin Piltan, M. Mousavi, A. Khajeh, M. R. Rashidian, "Intelligent Robust Feed-forward Fuzzy Feedback Linearization Estimation of PID Control with Application to Continuum Robot", International Journal of Information Engineering and Electronic Business, 5(1), 2013.

[28] Farzin Piltan, N. Sulaiman \& I.AsadiTalooki, "Evolutionary Design on-line Sliding Fuzzy Gain Scheduling Sliding Mode Algorithm: Applied to Internal Combustion Engine", International Journal of Engineering Science and Technology, 3 (10):7301-7308, 2011.

[29] Farzin Piltan, Nasri B Sulaiman, Iraj Asadi Talooki \& Payman Ferdosali, ”Designing On-Line Tunable Gain Fuzzy Sliding Mode Controller Using Sliding Mode Fuzzy Algorithm: Applied to Internal Combustion Engine" World Applied Science Journal (WASJ), 15 (3): 422-428, 2011.

[30] Farzin Piltan, M.J. Rafaati, F. Khazaeni, A. Hosainpour, S. Soltani, “A Design High Impact Lyapunov Fuzzy PD-Plus-Gravity Controller with Application to Rigid Manipulator", International Journal of Information Engineering and Electronic Business, 5(1), 2013.

[31] A. Jalali, Farzin Piltan, A. Gavahian, M. Jalali, M. Adibi, "Model-Free Adaptive Fuzzy Sliding Mode Controller Optimized by Particle Swarm for Robot manipulator", International Journal of Information Engineering and Electronic Business, 5(1), 2013.

[32] Farzin Piltan, N. Sulaiman, Payman Ferdosali, Mehdi Rashidi \& Zahra Tajpeikar, "Adaptive MIMO Fuzzy Compensate Fuzzy Sliding Mode Algorithm: Applied to Second Order Nonlinear System", International Journal of Engineering, 5 (5): 380-398, 2011.

[33] Farzin Piltan, N. Sulaiman, Hajar Nasiri, Sadeq Allahdadi \& Mohammad A. Bairami, "Novel Robot Manipulator Adaptive Artificial Control: Design a Novel SISO Adaptive Fuzzy Sliding Algorithm Inverse Dynamic Like Method", International Journal of Engineering, 5 (5): 399418, 2011.

[34] Farzin Piltan, N. Sulaiman, Sadeq Allahdadi, Mohammadali Dialame \& Abbas Zare, "Position Control of Robot Manipulator: Design a Novel SISO Adaptive Sliding Mode Fuzzy PD Fuzzy Sliding Mode Control", International Journal of Artificial Intelligence and Expert System, 2 (5):208-228, 2011.

[35] M. M. Ebrahimit Farzin Piltan, M. Bazregar and A.R. Nabaee "Intelligent Robust Fuzzy-Parallel 
Optimization Control of a Continuum Robot Manipulator", International Journal of Control and Automation, 6(3), 2013.

[36] Farzin Piltan, M.A. Bairami, F. Aghayari, M.R. Rashidian, "Stable Fuzzy PD Control with Parallel Sliding Mode Compensation with Application to Rigid Manipulator", International Journal of Information Technology and Computer Science, $5(7), 2013$

[37] Farzin Piltan, N. Sulaiman, Samaneh Roosta, Atefeh Gavahian \& Samira Soltani, "Evolutionary Design of Backstepping Artificial Sliding Mode Based Position Algorithm: Applied to Robot Manipulator", International Journal of Engineering, 5 (5):419-434, 2011.

[38] Farzin Piltan, N. Sulaiman, Amin Jalali, Sobhan Siamak \& Iman Nazari, "Control of Robot Manipulator: Design a Novel Tuning MIMO Fuzzy Backstepping Adaptive Based Fuzzy Estimator Variable Structure Control", International Journal of Control and Automation, 4 (4):91-110, 2011.

[39] Farzin Piltan, N. Sulaiman, Atefeh Gavahian, Samaneh Roosta \& Samira Soltani, "On line Tuning Premise and Consequence FIS: Design Fuzzy Adaptive Fuzzy Sliding Mode Controller Based on Lyaponuv Theory", International Journal of Robotics and Automation, 2 (5):381-400, 2011.

[40] Farzin Piltan, N. Sulaiman, Samira Soltani, Samaneh Roosta \& Atefeh Gavahian, "Artificial Chattering Free on-line Fuzzy Sliding Mode Algorithm for Uncertain System: Applied in Robot Manipulator", International Journal of Engineering, 5 (5):360-379, 2011.

[41] Farzin Piltan, F. ShahryarZadeh ,M. Mansoorzadeh ,M. kamgari, S. Zare, "Robust Fuzzy PD Method with Parallel Computed Fuel Ratio Estimation Applied to Automotive Engine "International Journal of Intelligent Systems and Applications, 5(8), 2013.

[42] Farzin Piltan, Sadeq Allahdadi, Mohammad A.Bairami \& Hajar Nasiri, "Design Auto Adjust Sliding Surface Slope: Applied to Robot Manipulator", International Journal of Robotics and Automation, 3 (1):27-44, 2011.

[43] Farzin Piltan, Mohammadali Dialame, Abbas Zare \& Ali Badri, "Design Novel Lookup Table Changed Auto Tuning FSMC:Applied to Robot Manipulator", International Journal of Engineering, 6 (1):25-41, 2012.

[44] Farzin Piltan, M. Keshavarz, A. Badri \& A. Zargari, "Design Novel Nonlinear Controller Applied to RobotManipulator: Design New Feedback Linearization Fuzzy Controller with Minimum Rule Base Tuning Method",
International Journal of Robotics and Automation, 3 (1):1-12, 2012.

[45] Farzin Piltan, Mohammad A.Bairami, Farid Aghayari \& Sadeq Allahdadi, "Design Adaptive Artificial Inverse Dynamic Controller: Design Sliding Mode Fuzzy Adaptive New Inverse Dynamic Fuzzy Controller", International Journal of Robotics and Automation, (1):13-26, 2012.

[46] Farzin Piltan, Sadeq Allahdadi, Mohammad A.Bairami \& Hajar Nasiri, "Design Auto Adjust Sliding Surface Slope: Applied to Robot Manipulator", International Journal of Robotics and Automation, 3 (1):27-44, 2012.

[47] Farzin Piltan, F. Aghayari, M. Rashidian \& M. Shamsodini, "A New Estimate Sliding Mode Fuzzy Controller for RoboticManipulator", International Journal of Robotics and Automation, 3 (1):45-60, 2012

[48] Farzin Piltan, Iman Nazari, Sobhan Siamak, Payman Ferdosali, "Methodology of FPGA-Based Mathematical error-Based Tuning Sliding Mode Controller", International Journal of Control and Automation, 5(1), 89-118, 2012.

[49] Farzin Piltan, Bamdad Boroomand, Arman Jahed \& Hossein Rezaie, "Methodology of Mathematical Error-Based Tuning Sliding Mode Controller", International Journal of Engineering, 6 (2):96-117, 2012.

[50] Farzin Piltan, S. Emamzadeh, Z. Hivand, F. Shahriyari \& Mina Mirazaei. " PUMA-560 Robot Manipulator Position Sliding Mode Control Methods Using MATLAB/SIMULINK and Their Integration into Graduate/Undergraduate Nonlinear Control, Robotics and MATLAB Courses", International Journal of Robotics and Automation, 3(3):106-150, 2012.

[51] Farzin Piltan, A. Hosainpour, E. Mazlomian, M.Shamsodini, M.H Yarmahmoudi. "Online Tuning Chattering Free Sliding Mode Fuzzy Control Design: Lyapunov Approach", International Journal of Robotics and Automation, 3(3):77-105, 2012.

[52] Farzin Piltan, R. Bayat, F. Aghayari, B. Boroomand. "Design Error-Based Linear ModelFree Evaluation Performance Computed Torque Controller", International Journal of Robotics and Automation, 3(3):151-166, 2012.

[53] Farzin Piltan, J. Meigolinedjad, S. Mehrara, S. Rahmdel. "Evaluation Performance of 2nd Order Nonlinear System: Baseline Control Tunable Gain Sliding Mode Methodology", International Journal of Robotics and Automation, 3(3): 192-211, 2012.

[54] Farzin Piltan, Mina Mirzaei, Forouzan Shahriari, Iman Nazari, Sara Emamzadeh, "Design Baseline 
Computed Torque Controller", International Journal of Engineering, 6(3): 129-141, 2012.

[55] Farzin Piltan, Sajad Rahmdel, Saleh Mehrara, Reza Bayat , "Sliding Mode Methodology Vs. Computed Torque Methodology Using MATLAB/SIMULINK and Their Integration into Graduate Nonlinear Control Courses" , International Journal of Engineering, 6(3): 142-177, 2012.

[56] Farzin Piltan , M.H. Yarmahmoudi, M. Shamsodini, E.Mazlomian, A.Hosainpour. "PUMA-560 Robot Manipulator Position Computed Torque Control Methods Using MATLAB/SIMULINK and Their Integration into Graduate Nonlinear Control and MATLAB Courses", International Journal of Robotics and Automation, 3(3): 167-191, 2012.

[57] Farzin Piltan, Hossein Rezaie, Bamdad Boroomand, Arman Jahed. "Design Robust Backstepping on-line Tuning Feedback Linearization Control Applied to IC Engine", International Journal of Advance Science and Technology, 11:40-22, 2012.

[58] Farzin Piltan, S. Siamak, M.A. Bairami and I. Nazari. "Gradient Descent Optimal Chattering Free Sliding Mode Fuzzy Control Design: Lyapunov Approach", International Journal of Advanced Science and Technology, 43: 73-90, 2012.

[59] Farzin Piltan, M.R. Rashidian, M. Shamsodini and S. Allahdadi." Effect of Rule Base on the FuzzyBased Tuning Fuzzy Sliding Mode Controller: Applied to 2nd Order Nonlinear System", International Journal of Advanced Science and Technology, 46:39-70, 2012.

[60] Farzin Piltan, A. Jahed, H. Rezaie and B. Boroomand. "Methodology of Robust Linear Online High Speed Tuning for Stable Sliding Mode Controller: Applied to Nonlinear System", International Journal of Control and Automation, 5(3): 217-236, 2012.

[61] Farzin Piltan, R. Bayat, S. Mehara and J. Meigolinedjad. "GDO Artificial IntelligenceBased Switching PID Baseline Feedback Linearization Method: Controlled PUMA Workspace", International Journal of Information Engineering and Electronic Business, 5: 17-26, 2012.

[62] Farzin Piltan, B. Boroomand, A. Jahed and H. Rezaie. "Performance-Based Adaptive Gradient Descent Optimal Coefficient Fuzzy Sliding Mode Methodology", International Journal of Intelligent Systems and Applications, 11: 40-52 2012.

[63] Farzin Piltan, S. Mehrara, R. Bayat and S. Rahmdel. " Design New Control Methodology of Industrial Robot Manipulator: Sliding Mode
Baseline Methodology", International Journal of Hybrid Information Technology, 5(4):41-54, 2012.

[64] AH Aryanfar, MR Khammar, Farzin Piltan, "Design a robust self-tuning fuzzy sliding mode control for second order systems", International Journal of Engineering Science REsearch, 3(4): 711-717, 2012.

[65] Farzin Piltan, Shahnaz Tayebi Haghighi, "Design Gradient Descent Optimal Sliding Mode Control of Continuum Robots", International Journal of Robotics and Automation, 1(4): 175-189, 2012.

[66] Farzin Piltan, A. Nabaee, M.M. Ebrahimi, M. Bazregar, "Design Robust Fuzzy Sliding Mode Control Technique for Robot Manipulator Systems with Modeling Uncertainties", International Journal of Information Technology and Computer Science, 5(8), 2013.

[67] Farzin Piltan, M. Akbari, M. Piran , M. Bazregar. "Design Model Free Switching Gain Scheduling Baseline Controller with Application to Automotive Engine", International Journal of Information Technology and Computer Science, 01:65-73, 2013.

[68] Farzin Piltan, M. Piran , M. Bazregar, M. Akbari, "Design High Impact Fuzzy Baseline Variable Structure Methodology to Artificial Adjust Fuel Ratio", International Journal of Intelligent Systems and Applications, 02: 59-70, 2013.

[69] Farzin Piltan, M. Mansoorzadeh, M. Akbari, S. Zare, F. ShahryarZadeh "Management of Environmental Pollution by Intelligent Control of Fuel in an Internal Combustion Engine“ Global Journal of Biodiversity Science And Management, 3(1), 2013

\section{Authors' Profiles}

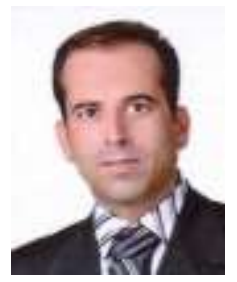

Mansour Bazregar is an industrial management researcher of research and development company SSP. Co. $\mathrm{He}$ is now pursuing his Master in industrial management. $\mathrm{He}$ is an expert Industrial and Quality Management in this company. His research activities deal with the IC engine control and supply chain management.

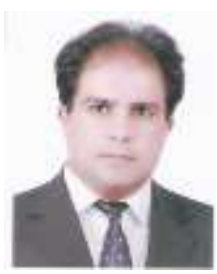

Farzin Piltan was born on 1975 , Shiraz, Iran. In 2004 he is jointed the research and development company, SSP Co, Shiraz, Iran. In addition to 7 textbooks, Farzin Piltan is the main author of more than 80 scientific papers in refereed journals. He is editorial board of international journal of control and 
automation (IJCA), editorial board of International Journal of Intelligent System and Applications (IJISA), editorial board of IAES international journal of robotics and automation, editorial board of International Journal of Reconfigurable and Embedded Systems and reviewer of (CSC) international journal of robotics and automation. His main areas of research interests are nonlinear control, artificial control system and applied to FPGA, robotics and artificial nonlinear control and IC engine modelling and control.

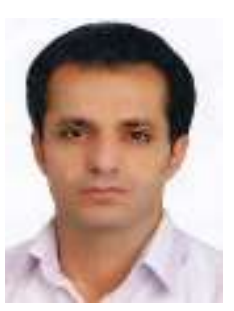

Mehdi Akbari is an industrial management researcher of research and development company SSP. Co. $\mathrm{He}$ is now pursuing his Master in industrial management. $\mathrm{He}$ is an expert Industrial and Quality Management in this company. His research activities deal with the IC engine control, artificial intelligence and supply chain management.

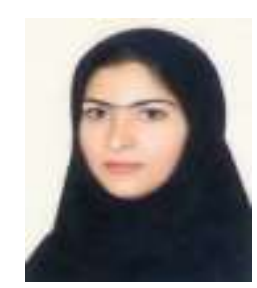

Mojdeh Piran is an industrial management researcher of research and development company SSP. Co. She is now pursuing her Master in industrial management. She is an expert Industrial and Quality Management in this company. Her research activities deal with the IC engine control, artificial intelligence and expert system.

How to cite this paper: Mansour Bazregar, Farzin Piltan, Mehdi Akbari, Mojdeh Piran,"Management of Automotive Engine Based on Stable Fuzzy Technique with Parallel Sliding Mode Optimization", International Journal of Information Technology and Computer Science(IJITCS), vol.6, no.1, pp.101-107, 2014. DOI: 10.5815/ijitcs.2014.01.12 